\title{
Oficina dos Perigos da Associação Entre Multitarefa, Álcool e Direção: Experiência em um Ambiente Não-Formal
}

\section{Workshop on the Dangers of the Association Among Multitasking, Alcohol and Driving: Experience in a Non-Formal Environment}

\author{
Ohana Turcato Macacare ${ }^{\mathrm{a}}$; Renata Waner Mariquito ${ }^{\mathrm{b}}$; Ana Flávia Guidi Mengato ${ }^{\mathrm{b}}$; Paulo Evandro Frattonye \\ Roberta Ekuni*c
}

\author{
${ }^{\text {a} F a c u l d a d e ~ d e ~ M e d i c i n a ~ d e ~ R i b e i r a ̃ o ~ P r e t o . ~ S P, ~ B r a s i l . ~}$

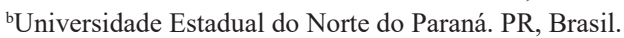 \\ 'Universidade Estadual do Norte do Paraná, Programa de Pós-Graduação em Educação. PR, Brasil. \\ *E-mail de contato: robertaekuni@uenp.edu.br
}

\begin{abstract}
Resumo
Multitarefa com mídias é um fenômeno no qual são realizadas mais de uma tarefa ao mesmo tempo, sendo pelo menos uma das atividades com aparelhos eletrônicos. Esta prática impacta, negativamente, várias situações do cotidiano. Muitas vezes, pode-se associá-la ao ato de dirigir e até mesmo envolver o consumo de bebida alcoólica, tornando potencialmente uma combinação fatal. Assim, os potenciais riscos associados a estas práticas devem ser divulgados e a extensão universitária pode contribuir promovendo ações de Divulgação Científica. Dessa forma, o objetivo do presente trabalho é relatar uma ação extensionista realizada por meio de uma oficina em um ambiente não-formal de educação. A oficina focava na divulgação dos riscos ligados a Multitarefa com Mídia, direção e consumo de álcool. Para isso, duas ações foram combinadas: a primeira abordou o tema Multitarefa com Mídia e a segunda associou a prática de Multitarefa com Mídia, direção e consumo de álcool. Ambas oficinas associaram conteúdos teóricos com atividades práticas. Como resultado, participaram destas ações alunos da Educação Básica, Educação Básica na modalidade Educação Especial e comunidade em geral. Os visitantes vivenciaram simulações, experimentos e puderam refletir sobre os prejuízos em potencial da combinação multitarefa, álcool e direção. O feedback dos visitantes foi positivo.
\end{abstract}

Palavras-chave: Multitarefa. Extensão Universitária. Educação Não-Formal.

\begin{abstract}
Media Multitasking is a phenomenon in which more than one task is performed simultaneously, being one of the activities with electronic devices. This practice negatively impacts several everyday situations. It can often be associated with driving and even involve drinking alcohol, potentially making it a fatal combination. Thus, the potential risks associated with these practices must be disclosed, and university extension can contribute by promoting Scientific Dissemination actions. Thus, this paper aims to report an extension action through a workshop in a nonformal educational environment. The workshop focused on disclosing the risks related to Multitasking with Media, management, and alcohol consumption. For this, two actions were combined: the first approached the topic of Multitasking with Media, and the second associated the practice of Multitasking with Media, driving, and alcohol consumption. Both workshops associated theoretical content with practical activities. As a result, students from Basic Education, Basic Education in the Special Education modality, and the community, in general, participated in these actions. Visitors experienced simulations, experiments, and reflected on the potential losses from the combination of multitasking, alcohol, and driving. The feedback from visitors was positive.
\end{abstract}

Keywords: Multitasking. University Extension. Non-Formal Education.

\section{Introdução}

Multitarefa significa executar várias tarefas ao mesmo tempo (PASHLER, 1994), por exemplo, cozinhar enquanto conversa com outra pessoa. Em função do desenvolvimento tecnológico, um fenômeno mais atual é a Multitarefa com Mídias (do inglês - Media Multitasking), ou seja, realizar duas ou mais tarefas simultaneamente, na qual em pelo menos uma delas se utiliza um dispositivo eletrônico (OPHIR; NASS; WAGNER, 2009; RALPH et al., 2013).

Pesquisas mostram que realizar múltiplas tarefas, simultaneamente, acarreta em vários problemas cognitivos, acadêmicos e socioemocionais, como problemas na memória de trabalho e na atenção (OPHIR; NASS; WAGNER, 2009; VAN DER SCHUUR et al., 2015), principalmente, no momento de realizar tarefas que necessitam de uma atenção sustentada (RALPH et al., 2015). Por exemplo, alunos que utilizam aparelhos eletrônicos no momento do estudo, tanto dentro quanto fora de sala de aula, apresentam piores desempenhos na recordação do conteúdo quando comparados com alunos que não realizaram Multitarefa com Mídias nesta situação (JACOBSEN; FORSTE, 2011; GINGERICH; LINEWEAVER, 2014; DEMIRBILEK; TALAN, 2018). Este impacto negativo no aprendizado também é observado, quando são utilizadas mídias de forma concomitante com o dever de casa (MARTÍN-PERPIÑÁ; POCH; CERRATO, 2019).

Uma possível explicação para os diversos prejuízos relacionados com a prática de Multitarefa se deve à atenção dividida, a qual prediz que o processamento de múltiplas informações é realizado de forma imperfeita e, dessa forma, informações importantes podem ser perdidas (POSNER, 1990). 
Isso pode explicar o motivo de que uma das principais causas de acidentes de trânsito é a utilização de aparelhos eletrônicos, enquanto o motorista está dirigindo (STRAYER; WATSON; DREWS, 2011; KIM et al., 2017). A utilização de mídias nesta situação desvia o foco atencional do motorista, que deveria estar concentrado no ato de dirigir e essa desatenção pode acarretar em um atraso na reação do motorista, o que pode levar a um acidente (STRAYER; WATSON; DREWS, 2011).

Outro problema no momento de dirigir, é causado pelo consumo de bebidas alcoólicas por parte dos motoristas (DAMACENA et al., 2016; VALEN et al., 2019), podendo influenciar, negativamente, o funcionamento da atenção (MARTIN et al., 2013; MIAO et al., 2017). Além disto, o consumo de álcool pode estar relacionado a um comportamento de condução arriscada por parte do motorista, como uma maior aceleração e frenagens abruptas que pode levar a um acidente (YADAV; VELAGA, 2019).

A prática de Multitarefa com Mídias é algo comum (RALPH et al., 2015) e a utilização de mídias pelo motorista está associada a uma das principais causas de acidente de trânsito (STRAYER; WATSON; DREWS, 2011; KIM et al., 2017). Associado a essa dupla, também há o consumo de álcool, diretamente ligado a acidentes de trânsito (VALEN et al., 2019).

Tendo em vista esses problemas, realizar divulgações científicas que abordam essas temáticas em conjunto é de extrema importância. A divulgação científica é uma forma de descentralizar o conhecimento científico do mundo acadêmico, gerando oportunidades para que a comunidade não científica tenha o acesso a este conhecimento (VARGAS et al., 2014), seja por meio de divulgações midiáticas, em geral, ou por meio da inclusão destes em locais como os museus (CAVALCANTI; PERSECHINI, 2019).

Uma forma de se divulgar a ciência é por meio da extensão universitária, e essa por sua vez faz parte do tripé que sustenta uma Universidade, junto com a pesquisa e o ensino (FORPROEX, 2012). A Extensão apresenta como características centrais a disseminação do conhecimento acadêmico, prestações de serviços para a comunidade e a difusão de conhecimento e cultura, sendo assim uma forma de conectar a Universidade com a sociedade (FORPROEX, 2012).

O Programa de Extensão Grupo de Estudos em Neurociências (GEN) (EKUNI et al., 2014) organiza, desde 2014, um evento extensionista no estilo exposição de ciências, cujo objetivo é divulgação científica, chamado "Conhecendo o Cérebro" (e.g. SOUZA et al., 2016). No ano de 2019, o "Conhecendo o Cérebro" apresentou como temática central "Bioeconomia: diversidade e riqueza para o desenvolvimento sustentável" seguindo a temática da "Semana Nacional da Ciência e Tecnologia". O evento em questão apresentou nove estandes que abordavam diferentes temas, entre esses o "Estande de Multitarefa" e "Se Beber Não Dirija" os quais serão relatados neste artigo. Em edições anteriores do evento foram abordados tanto a temática álcool e direção (ROCHA et al., 2019a) quanto prevenção de lesão crânio encefálica em acidentes de trânsito (CARVALHO et al., 2020) e a partir do relato dos visitantes, pode-se perceber o quanto estas temáticas são de interesse e curiosidade destes.

A combinação entre a prática de Multitarefa, o consumo de álcool e a direção irresponsável afetam no cotidiano, e boa parte do público-alvo do evento são adolescentes, ou seja, um público vulnerável a essa mistura perigosa (GICQUEL et al., 2017). Desse modo, o objetivo dos estantes relatados no presente artigo foram popularizar os conhecimentos científicos, que versam a realização de Multitarefa, o consumo de bebidas alcoólicas e, os possíveis riscos que estas práticas podem causar, principalmente, quando associadas à direção de automóveis.

\section{Material e Métodos}

\subsection{O público-alvo}

Essa ação foi direcionada aos estudantes da Educação Básica de escolas públicas e privadas, bem como alunos da Educação Básica na modalidade Educação Especial (Associação dos Pais e Amigos dos Excepcionais - APAE). A ação contemplou as cidades de Abatiá, Bandeirantes, Japira e Santa Mariana. Além disso, recebeu visitas de docentes e discentes da UENP, bem como do público em geral.

\subsection{A ação extensionista}

A ação extensionista ocorreu no Auditório Thomas Nicoletti, localizado na Universidade Estadual do Norte do Paraná, Campus Luiz Meneghel, na cidade de Bandeirantes, Paraná, no dia 22 de outubro de 2019. Sua divulgação ocorreu por meio das redes sociais como Facebook, Whatsapp e Instagram. A ação foi gratuita e englobou vários estandes, cada um com seus objetivos e coordenadores específicos.

\subsection{Procedimento}

A ação extensionista aqui descrita foi distribuída em duas etapas:

\section{I) $O$ estande de Multitarefa}

No estande de Multitarefa (Figura 1A), as monitoras primeiramente explicaram o que significa realizar multitarefa com base na literatura citada na introdução. Posteriormente, questionaram a opinião dos visitantes a respeito do assunto, se consideravam que eram bons ou não em realizar mais de uma tarefa por vez. Após o breve debate, os visitantes assistiram um vídeo desafio de 3 minutos de duração ("Você é multitarefas?" Canal do Valentim, 2014) (Figura 2A). No vídeo, havia um desafio no qual o locutor apresentava uma série de palavras que o visitante deveria lembrar ao final, ao mesmo tempo em deveriam analisar uma imagem de roldanas estáticas visando descobrir para qual lado o ponteiro da última roldana iria 
apontar após a primeira roldana girar, se para a ovelha ou para o porco. Visto que a execução desta tarefa poderia ser de difícil entendimento para certos visitantes, assim como em outras atividades propostas, adaptava-se a linguagem e a atividade de acordo com a faixa-etária.

Figura 1 - A) Participação dos alunos de Ensino Médio no estande de "Multitarefas"; B) monitora do estande de "Multitarefa" adaptando a linguagem do vídeo utilizado para explicar os conceitos para uma criança; C) monitorar explicando os conceitos apresentados no estande "Se beber não dirija" e D) visitante do evento realizando a dinâmica de simulação de embriaguez.

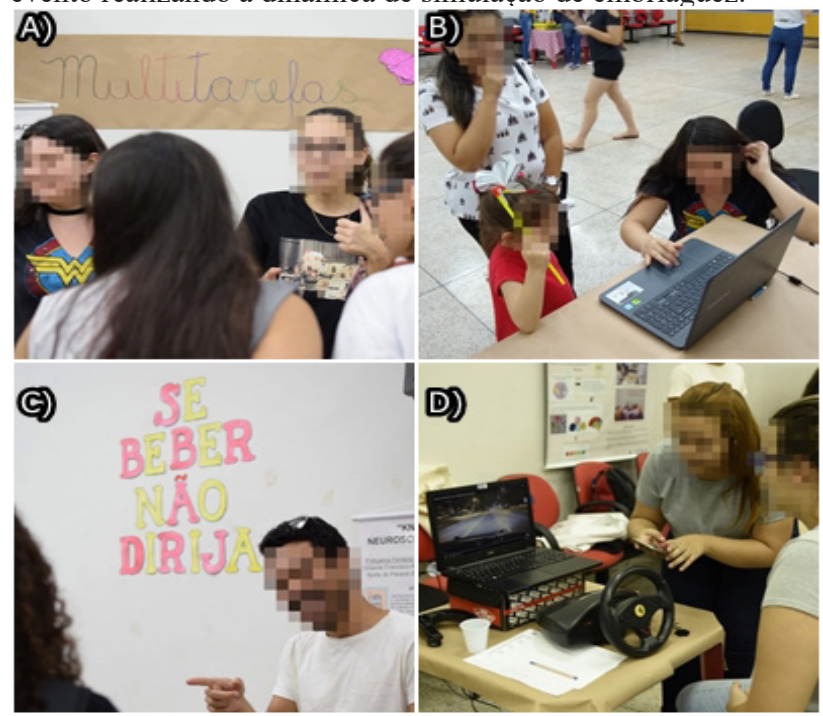

Fonte: os autores.

Figura 2 - A) Computador usado para projetar o vídeo "Você é multitarefas?"; B) Espelho utilizado no "Estande de Multitarefa" para evidenciar o fenômeno da cegueira sacádica.
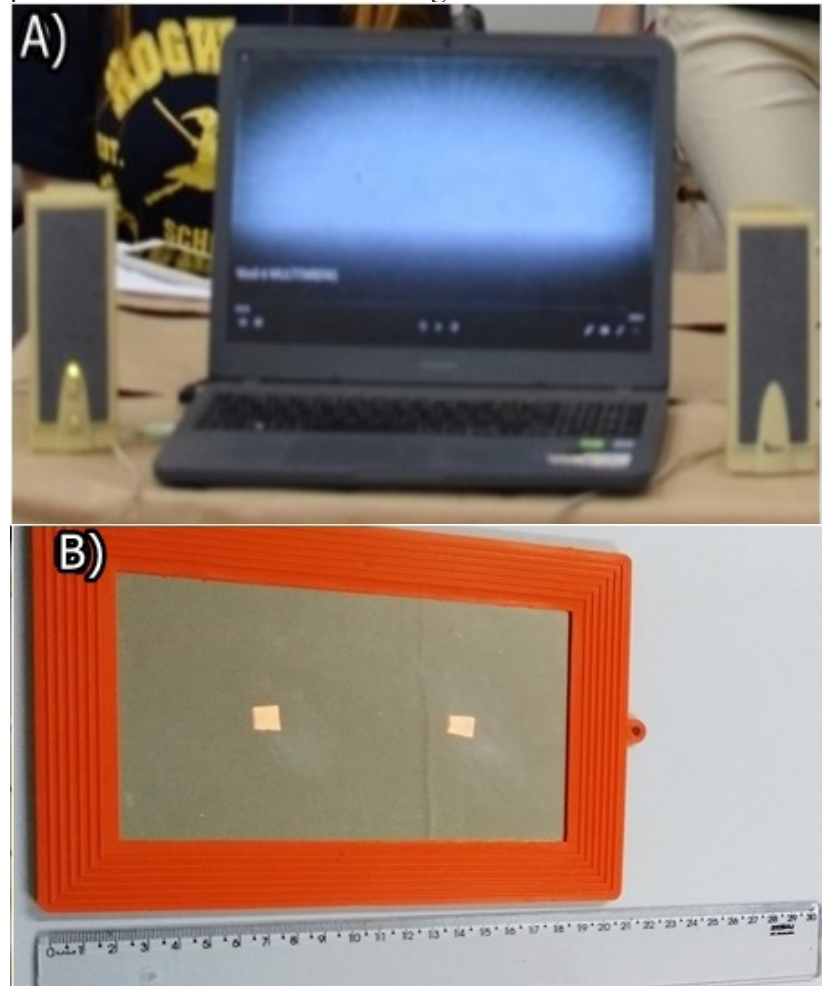

Fonte: Os autores

Ao final do vídeo, os visitantes respondiam a quantidade de palavras que se lembravam, além de verificar se acertaram para qual dos animais o ponteiro virou. Posteriormente, o locutor do vídeo informou aos ouvintes que, durante a atividade, houve modificações no vídeo, que provavelmente não perceberam, mostrando como a Multitarefa pode afetar a atenção. Usando isso como gancho, as monitoras explicaram como o uso de Multitarefa no trânsito pode ser prejudicial, principalmente, se utilizado junto com aparelhos eletrônicos, como o celular. Para isso, foi realizada uma prática, a fim de exemplificar a cegueira sacádica, definida como um momento de poucos milissegundos no qual a percepção visual é comprometida quando há uma mudança na direção do olhar (PURVES et al., 2018). Nessa dinâmica, o visitante deveria segurar um espelho e olhar de um ponto, marcado com caneta no próprio espelho, para outro a uma distância de, mais ou menos, $8 \mathrm{~cm}$ (Figura 2B). Após isso, questionava se eles eram capazes de ver a movimentação dos seus próprios olhos. Como não é possível, eles respondiam que não. Entretanto, os demais visitantes podiam confirmar que os olhos do visitante no espelho se moviam, pois eles ficavam observando a tarefa. Desse modo, era possível exemplificar como isso pode atrapalhar na direção, pois apenas uma mudança na direção do olhar, por exemplo, olhar no telefone, já pode deixar o motorista "cego", por tempo suficiente, para não ver algo em sua frente e se acidentar.

Ao final da explicação, como nos anos anteriores, os visitantes que desejassem, forneciam um feedback em uma tabela, ao lado da idade e sexo, marcando qual sua reação para com o estande utilizando uma escala Likert de emojis (ROCHA et al., 2019a).

\section{II) Estande Álcool e Direção}

Visando demonstrar os perigos de beber e dirigir, bem como pensando no fenômeno Multitarefa, o presente estande (Figura 1C) foi modificado de Rocha et al. (2019a). Entretanto, continuou utilizando os óculos que simulam a embriaguez (3bScientific).

Para a execução do presente estande, uma mesa simulava um bar (com uma garrafa, um copo e uma chave de carro), no qual o visitante simulava estar se embriagando. Então, colocavam os óculos, pegavam a chave e simulavam sair do bar. Então, se direcionavam para outra mesa. O caminho que levava uma mesa para outra era um espaço de 1,5 metros de distância, marcado em linha reta com fita colante branca. O visitante tentava andar em linha reta, entretanto, uma vez que estava com os óculos simulador de embriaguez, sua percepção ficava distorcida e o mesmo podia perceber o quanto era difícil chegar até a segunda mesa. Nessa, havia um simulador de direção para Videogame e um notebook, utilizado como tela de vídeo para o jogo, no qual o visitante simulava dirigir, ao mesmo tempo, em que deveria ver uma mensagem que recebia no celular, que ficava ao lado do volante visitante (Figura 1D).

Ao final da dinâmica, foi explicado como a visão e outros processos sensoriais são afetados pelo consumo de álcool 
(BACHETTI; FUKUSIMA; QUAGLIA, 2017). Além disso, reforçava a dificuldade de dirigir e ver o celular, o que pode ser remetido à dinâmica dos movimentos sacádicos dos olhos do estande anterior. Os monitores explicaram os possíveis problemas fisiológicos e diversas patologias decorrentes do excesso de bebida alcoólica, como a esteatose hepática (gordura no fígado), pancreatite, hepatite, doenças cardíacas, instabilidade muscular, atrofia do cerebelo, neuropatia periférica, distúrbios de coordenação, delírios, alterações de humor, demência, gastrite, úlceras (PELICIONI et al., 2017; COSTA; AGUIAR; SOUTO, 2017; MONCALEANO; BRANDS, 2019).

\subsection{Análise dos resultados}

Para analisar os resultados, os autores, que também foram monitores do evento, se valeram de sua percepção (subjetividade) mediante a observação participante no evento em questão, ou seja, com exceção da escala Likert de emojis que trata da opinião dos visitantes a respeito do evento, os demais dados partiram da observação participante dos monitores.

\section{Resultados e Discussão}

Participaram do evento 335 visitantes, desses 66 eram alunos da Educação Básica na modalidade Educação Especial. No total, 79 visitantes (média de idade de 19,03 \pm 7,81 anos; $70 \%$ mulheres) avaliaram o estande de Multitarefa.

Durante a primeira prática realizada, neste estande, foi observado que as pessoas não conseguiam lembrar de muitas palavras, embora algumas pessoas acertaram para qual animal o ponteiro indicou, o que havia uma chance de $50 \%$ de acertar ao acaso, já que só havia duas opções. Entretanto, foram raras as pessoas que perceberam as mudanças que ocorrerem na tela, e, as poucas que notaram, relataram no máximo duas mudanças.

Um adendo importante ao se discutir sobre essa prática foi que, no início, quando se questionava se os visitantes eram bons em realizar Multitarefa, diversas mulheres diziam que sim, usando o exemplo de serem donas de casa e relataram situações cotidianas, em que realizavam várias tarefas de forma concomitantemente. Corroborando com os relatos feitos por essas visitantes, a literatura indica que este estereótipo social, de que mulheres são melhores em Multitarefa do que os homens, vem da crença de que elas conseguem gerenciar, com uma suposta eficiência, situações como realizar trabalhos domésticos ao mesmo tempo em que cuidam dos filhos (SZAMEITAT et al., 2015). Entretanto, mulheres não realizam múltiplas tarefas por apresentar mais habilidade para isso, visto que em testes que envolvem Multitarefa tanto homens quanto mulheres apresentam um desempenho ruim, mas sim por serem socialmente pressionadas para isso ( $\mathrm{HIRSCH}$; KOCH; KARBACH, 2019).

Além desse relato, crianças e adolescentes, que passaram pelo estande, proferiram outros relatos interessante. Por exemplo, uns afirmavam fazer o uso de aparelho celular em vários momentos como durante o estudo, dentro e fora de sala de aula, e que não percebiam o quanto isso era prejudicial até se depararem com as informações ditas no estande.

A segunda prática também se mostrou eficaz para que os visitantes entendessem o conceito de cegueira sacádica, e os mesmos relataram que realmente não conseguiam ver o trajeto que seus olhos faziam de um ponto ao outro. Alguns ficavam surpresos quando os colegas diziam que o olho se mexia de fato, porque eles não conseguiam ver.

$\mathrm{Na}$ escala Likert de emojis, as monitoras notaram que alguns alunos mais novos, principalmente da Educação Básica na modalidade Educação Especial do sexo masculino, não queriam assinalar a opção do emoji com os olhos de coração na escala, pois poderia ser considerado "algo de menina". Isso pode ser um indicativo de uma dificuldade de expressão dos sentimentos por parte do sexo masculino em função de a sociedade culturalmente machista (NIGRO; BARACAT, 2018). Para eventos futuros, a organização do evento pode repensar na escala Likert de emojis devido a esse feedback. Ainda assim, os visitantes relataram que o estande foi uma experiência muito gratificante, que conseguiram aprender de forma prática os conceitos que nem mesmo paravam para analisar no dia-a-dia.

No total, $10,13 \%$ das pessoas que responderam o feedback marcaram "Gostei" na escala, enquanto os $89,87 \%$ restantes marcaram "Gostei Muito", resultados estes muito próximos a encontrados em eventos anteriores (ROCHA et al., 2019a, 2019b), o que demonstra que o evento mantém a característica de popularizar a ciência de uma forma agradável ao público.

Já em relação a segunda parte, no estande de Álcool e Direção, 61 pessoas (idade média de 16,81 $\pm 3,52$ anos; $53 \%$ mulheres) responderam o feedback. Na primeira simulação, alguns visitantes relataram que, ao colocarem os óculos simulador de embriaguez (3bScientific), a sensação visual era a mesma de quando bebem. Entretanto, observase que a maioria dos visitantes eram menores de 18 anos e a legislação brasileira reconhece por maior de idade e direito a habilitação de condutor, a idade superior a 18 anos. Uma parte dos visitantes relataram que já estavam tão acostumados com o álcool que nem sentiam a diferença, ou quando o tipo de alteração causada pelos óculos ocorre, já paravam de beber, para quando chegar em casa, os pais não percebessem que houve ingestão de álcool.

$\mathrm{Na}$ segunda simulação, percebe-se que alguns visitantes tiveram dificuldade para se mantem atento, seguir normas básicas do trânsito, e ter reflexo para evitar acidentes. Ao final da segunda fase, os visitantes puderam relacionar os estandes de Álcool e Direção com Multitarefa, mediante à dificuldade de estar utilizando os óculos simulador de embriaguez, tentar atender o celular e focar na pista. Assim, puderam perceber que essa tríade não combina, ou parafraseando a "gíria" relatada pelos adolescentes, não "dá match".

Em relação ao feedback, $3.27 \%$ dos visitantes marcaram 
a opção "Gostei" e 96.7\% marcaram a opção "Gostei Muito". Isso demonstra o agrado e satisfação do público em relação aos eventos de extensão e seu impacto. Os monitores do estande também relatam que a experiência foi muito gratificante, principalmente os que tiveram o primeiro contato com a extensão universitária por meio da participação na execução desses estandes, bem como o aprendizado horizontal proporcionado por este.

Uma limitação do presente trabalho é que, por se tratar de um evento em forma de exposição, não há uma ordem cronológica, ou seja, pode ser que alguns visitantes não tenham passado no primeiro estande para depois passar no segundo, ou que alguns não tenham participado em ambos. Outra limitação é que, por serem atividades rápidas, nem todos que passaram pelos estandes preencheram a tabela de feedback, desse modo, a avaliação dos estandes é apenas uma amostra da opinião dos visitantes do evento. Por fim, uma outra limitação é que se trata de uma ação pontual e rápida, em uma exposição. Trabalhos futuros poderiam avaliar sistematicamente se as presentes ações possuem efeito de longo-prazo.

\section{Conclusão}

Os eventos de extensão são cruciais para a formação acadêmica dos monitores, que devem se preparar para expor os conteúdos, havendo aprendizagem não só para os visitantes, mas também para os alunos. Ou seja, há benefício tanto para a sociedade quanto para os extensionistas.

Em relação ao estande de "Multitarefa", perceber-se que muitos visitantes relataram que realizam muitas tarefas ao mesmo tempo e que a percepção das pessoas quanto suas habilidades não condizem com a realidade. Desta forma, conscientizar a população sobre o quanto realizar múltiplas tarefas de forma concomitante é prejudicial é de extrema importância, principalmente para deixa-las a par dos possíveis riscos não só na direção, mas também nos estudos, visto que a maior parte do público foram estudantes da Educação Básica.

Em relação ao estande "Álcool e Direção", os relatos dos adolescentes nos levam a percepção de que muitos têm contato com a bebida antes da maioridade, o que é proibido por lei. Entretanto, os adolescentes puderam refletir sobre os perigos dessa combinação, mesmo de que forma pontual. Assim, nota-se a importância de levar esse estande para as escolas, aumentando o número de pessoas alcançadas.

\section{Agradecimentos}

À Universidade Estadual do Norte do Paraná - Campus Luiz Meneghel. À PROEC/UENP e à PROPG/UENP.

\section{Referências}

BACHETTI, L.S.; FUKUSIMA, S.S.; QUAGLIA, M.A.C. O efeito do álcool na percepção visuoespacial e na cognição do espaço. Psicol., Saúde Doenças, v. 18, n. 2, p.451-461, 2017.

CARVALHO, M.L. et al. Conhecer para previnir: a importância da extensão universitária na divulgação neurocientífica para prevenção de lesão cerebral. Conexão UEPG, v.16, 2020. https:// doi.org/10.5212/Rev.Conexao.v.16.13672.013

CAVALCANTI, C.C.B.; PERSECHINI, P.M. Museus de Ciência e a popularização do conhecimento no Brasil Museus de Ciência e a popularização do conhecimento no Brasil. Field Actions Scie. Rep.s, n.3, p.1-10, 2019.

COSTA, J.L.; AGUIAR, D.M.; SOUTO, Á. As consequências sociais, familiares, físicas e psíquicas do dependente de álcool. Rev. Nova Hileia, v.3, n.2, 2017.

DAMACENA, G. N. et al. Consumo abusivo de álcool e envolvimento em acidentes de trânsito na população brasileira, 2013. Ciênc. Saúde Coletiva, v.21, n.12, p.3777-3786, 2016.

DEMIRBILEK, M.; TALAN, T. The effect of social media multitasking on classroom performance. Active Learning in Higher Educ., v.19, n.2, p.117-129, 2018.

EKUNI, R. et al. Projeto de Extensão “ Grupo de Estudos em Neurociência ": divulgando neurociência e despertando vocações. Rev. Bras. Extensão Univers., v.5, n.2, p.55-59, 2014.

FORPROEX. Fórum de Pró-Reitores de Extensão das Universidades Públicas Brasileiras-FORPROEX. Disponível em: $<$ https://www.ufmg.br/proex/renex/images/documentos/201207-13-Politica-Nacional-de-Extensao>.

GICQUEL, L. et al. Description of various Factors Contributing to Traffic Accidents in Youth and Measures Proposed to Alleviate Recurrence. Front. Psychiatry, v. 8, p.1-10, 2017.

GINGERICH, A. C.; LINEWEAVER, T. T. OMG! Texting in Class 1/4 U Fail :( Empirical Evidence That Text Messaging During Class Disrupts Comprehension. Teaching Psychol., v.41, n.1, p.44-51, 2014.

HIRSCH, P.; KOCH, I.; KARBACH, J. Putting a stereotype to the test: The case of gender differences in multitasking costs in task-switching and dual-task situations. Plos One, v. 14, n. 8, p. $1-16,2019$.

JACOBSEN, W. C.; FORSTE, R. The Wired Generation: Academic and Social Outcomes of Electronic Media Use Among University Students. Cyberpsychol. v.14, n.5, p 275-280, 2011.

KIM, H.-J. et al. Accident risk associated with smartphone addiction: A study on university students in Korea. J. Behavioral Addict., v.6, n.4, p.699-707, 2017. doi: https://akjournals.com/ doi/10.1556/2006.6.2017.070.

MACACARE, O. T. et al. Ciência: alimento para o cérebro uma proposta multidisciplinar de popularização da ciência. Rev. Dialogos, v.22, n. 1, p.7-18, 2019.

MARTIN, T. L. et al. A review of alcohol-impaired driving: The role of blood alcohol concentration and complexity of the driving task. J. Forensic Scie., v. 58, n. 5, p. 1238-1250, 2013.

MARTÍN-PERPIÑÁ, M.M.; POCH, F.V.; CERRATO, S.M. Media multitasking impact in homework, executive function and academic performance in spanish adolescents. Psicothema, v.31, n.1, p. 81-87, 2019.

MIAO, L. et al. Effects of alcohol on drivers' perceptual ability and attention to concentration. Int. J. Contemp. Appl. Res., v.4, n. 8, p.42-49, 2017.

MONCALEANO, J. D.; BRANDS, B. Percepção de risco e direção sob efeitos do álcool e da maconha em estudantes universitários em um estudo multicéntrico: Colômbia. Texto Contexto Enferm., v.28, 2019. doi: https://doi.org/10.1590/1980$265 \mathrm{x}$-tce-cicad-24-28

NIGRO, I. S.; BARACAT J. Masculinidade: preciosa como 
diamante, fragil como cristal. Rev. Cient. Eletrônica Psicol., v. 30, p.4-19, 2018.

OPHIR, E.; NASS, C.; WAGNER, A. D. Cognitive control in media multitaskers. Proc. Nat. Acad. Scie., v.106, n.37, p.1558315587, 2009.

PASHLER, H. Dual-Task interference in simple tasks: data and theory. Psychol. Bull., v.116, n.2, p.220-244, 1994.

PELICIONI, M. et al. Perfil do consumo de álcool e prática do beber pesado episódico entre universitários brasileiros da área da saúde. J. Bras. Psiquiatr., v.66, n.3, p.150-156, 2017. doi: https:// doi.org/10.1590/0047-2085000000164.

POSNER, M. I. Hierarchical distributed networks in the neuropsychology of selective attention. Cog. Neuropsychol. Neurol., p.187-210, 1990.

PURVES, D. et al. Neurociências. São Paulo: Artmed, 2010.

RALPH, B.C. et al. Media multitasking and failures of attention in everyday life. Psychol. Res., v.78, n.5, p.661-669, 2013.

RALPH, B. et al. Media multitasking and behavioral measures of sustained attention. Attention, Perception Psychophysics, v.77, n.2, p.390-401, 2015.

ROCHA, M.B. et al. Se beber não dirija: popularizando os efeitos do álcool em um evento interativo. Rev. Extensão $U F M G$, v. 7, p.143-150, 2019a.

ROCHA, M. B. et al. Intervenção musicalizando: experimentação musical em uma ação extensionista. Rev. Bras. Extensão Univer., v.10, n.3, p.115-121, 2019b.

SOUZA, R.E. et al. "Conhecendo o cérebro": divulgando e despertando interesse na neurociência. Rev. Ciênc. Extensão, v.12, n.2, p.125-140, 2016.

STRAYER, D.L.; WATSON, J M.; DREWS, F. A. Cognitive distraction while multitasking in the automobile. Psychol. Learning Motiv., p.29-58, 2011. doi: 10.1016/B978-0-12-3855275.00002-4

SZAMEITAT, A. J. et al. "Women are better than men"-Public beliefs on gender differences and other aspects in multitasking. PLoS ONE, v.10, n.10, p.2-6, 2015.

VALEN, A. et al. Driver-related risk factors of fatal road traffic crashes associated with alcohol or drug impairment. Accident Anal. Prevention, v. 131, p. 191-199, 2019.

VAN DER SCHUUR, W. A. et al. The consequences of media multitasking for youth: A review. Comp. Hum. Behavior, v.53, p.204-215, 2015. doi: http://dx.doi.org/10.1016/j. chb.2015.06.035.

VARGAS, L.S. et al. Conhecendo o sistema nervoso: ações de divulgação e popularização da neurociência junto a estudantes da rede pública de Educação Básica. Ciênc. Cognição, v.19, n.2, p.233-241, 2014.

YADAV, A. K.; VELAGA, N. R. Effect of alcohol use on accelerating and braking behaviors of drivers. Traffic Injury Prev., v.20, n.4, p.353-358, 2019. doi: https://doi.org/10.1080/15389588.2019.1587167. 